\title{
Drug resistance profile and biofilm forming potential of Pseudomonas aeruginosa isolated from contact lenses in Karachi-Pakistan
}

Syed H Abidi ${ }^{1,2^{*}}$, Sikandar K Sherwani ${ }^{1,3}$, Tarrunum R Siddiquii ${ }^{4}$, Asma Bashir ${ }^{5}$ and Shahana U Kazmi ${ }^{1}$

\begin{abstract}
Background: The contaminated contact lens provides Pseudomonas aeruginosa an ideal site for attachment and biofilm production. Continuous contact of the eye to the biofilm-infested lens can lead to serious ocular diseases, such as keratitis (corneal ulcers). The biofilms also prevent effective penetration of the antibiotics, which increase the chances of antibiotic resistance.

Methods: For this study, 22 Pseudomonas aeruginosa isolates were obtained from 36 contact lenses and 14 contact lens protective fluid samples. These isolates were tested against eight commonly used antibiotics using Kirby-Bauer disk diffusion method. The biofilm forming potential of these isolates was also evaluated using various qualitative and quantitative techniques. Finally, a relationship between biofilm formation and antibiotic resistance was also examined.

Results: The isolates of Pseudomonas aeruginosa tested were found resistant to most of the antibiotics tested. Qualitative and quantitative biofilm analysis revealed that most of the isolates exhibited strong biofilm production. The biofilm production was significantly higher in isolates that were multi-drug resistant $(p<0.0001)$.

Conclusion: Our study indicates that multi-drug resistant, biofilm forming Pseudomonas aeruginosa isolates are mainly involved in contact lens associated infections. This appears to be the first report from Pakistan, which analyzes both antibiotic resistance profile and biofilm forming potential of Pseudomonas aeruginosa isolates from contact lens of the patients with contact lens associated infections.
\end{abstract}

Keywords: Antibiogram, Biofilm, Pseudomonas aeruginosa, Contact lens

\section{Background}

Pseudomonas aeruginosa is a clinically significant pathogen involved in several important infections, such as nosocomial, respiratory tract, urinary tract, burns, wound, and eye, etc. [1-4]. One of the hallmarks of Pseudomonal infection is its capability to adhere to and propagate on medical devices like catheters, contact lenses etc. The adherence is aided by several microbial factors, in which biofilm formation holds a key position [4,5]. Contact lens is one such devise that is frequently used for medical or cosmetic purpose. Improper handling and unhygienic use of

\footnotetext{
* Correspondence: m.haniabidi@gmail.com

${ }^{1}$ Immunology and Infectious Diseases Research Lab, Department of

Microbiology, University of Karachi, Karachi, Pakistan

${ }^{2}$ Department of Biological and Biomedical Sciences, Aga Khan University,

Stadium Road, Karachi 74800, Pakistan

Full list of author information is available at the end of the article
}

the contact lens allows pathogens, such as Pseudomonas aeruginosa, to adhere to and produce biofilm on the surface of the lens [6]. Continuous contact of the eye to the biofilminfested lens can result in serious eye infections such as keratitis (corneal ulcers) [7-10], which, if left untreated, can ultimately lead to vision loss. The improper management of these infections not only cause serious eye problems, but also increase the chances for antibiotic resistance [11].

In countries like Pakistan, contact lenses, of varying price and quality, can be purchased easily without any authentic prescription. Most of the users are unaware of proper hygienic practices, and as a result, they often face contact lens associated ophthalmic complications. In Pakistan, there are no significant details available about the burden of contact lens associated infection by Pseudomonas aeruginosa and antimicrobial resistance profile 
in such cases. In this study, we analyzed the antibiotic susceptibility/resistance profile and biofilm forming potential of Pseudomonas aeruginosa - isolated from patients with contact lens associated infections. Furthermore, we also examined the relationship between Pseudomonas aeruginosa biofilm forming potential and antibiotic resistance profile.

\section{Methods}

a) Sample collection, isolation and identification of cultures For this study, a total of 36 contact lenses, as well as 14 samples of contact lens protective fluid, were collected from Akhter Eye Hospital, Karachi-Pakistan. The contact lenses were aseptically immersed into Brain Heart Infusion (BHI) broth (Sigma-Aldrich) tubes and vortexed for 1 minute. Subsequently, the lenses were removed and the $\mathrm{BHI}$ broth tubes were incubated at $37^{\circ} \mathrm{C}$ for 24 hours. After incubation, a loop full of broth was inoculated on the Nutrient Agar (Sigma-Aldrich) plates and the plates were incubated at $37^{\circ} \mathrm{C}$ for 24 hours. For contact lens fluid samples, a loop full of liquid was directly inoculated and smeared on the Nutrient Agar (SigmaAldrich) plates and the plates were incubated at $37^{\circ} \mathrm{C}$ for 24 hours. The cultures were identified by using conventional and rapid biochemical tests (Quick test strip 24, DESTO, Pakistan).

\section{b) Antibiogram development}

Antibiotic susceptibility/resistance patterns of Pseudomonas aeruginosa to Vancomycin (VA), Erythromycin (E), Tetracycline (TE), Chloramphenicol (C), Ampicillin (AMP), Ofloxacin (OFX), Cephalexin (CL) and Gentamicin (CN) was examined by Kirby-Bauer method [12]. Briefly, 0.5 McFarland $\left(10^{8} \mathrm{cfu} / \mathrm{ml}\right)$ Pseudomonas aeruginosa inoculum was smeared on the Nutrient Agar plates, and abovementioned antibiotics discs were placed onto the agar. The plates were incubated at $37^{\circ} \mathrm{C}$ for $24-48$ hours, after which, the zone of inhibition was measured.

\section{c) Evaluation of biofilm forming potential}

The biofilm forming potential of Pseudomonas aeruginosa was evaluated by following methods: 1) Tube method, 2) Air Liquid Interface Assay, and 3) Microtitre-plate method.

\section{Tube method}

The tube method was performed in two different ways [13]. In the first experiment the cultures of Pseudomonas aeruginosa were inoculated in 3-5 ml Tryptone Soy Broth (TSB; Sigma-Aldrich) tubes and inoculated at $37^{\circ} \mathrm{C}$ for 48 hours. After incubation, the biofilm formation (dense matt formation) was observed at the air-liquid interface of the tubes. In the second experiment, cultures of Pseudomonas aeruginosa were inoculated in $3-5 \mathrm{ml}$
Tryptone Soy Broth (TSB; Sigma-Aldrich) tubes and incubated at $37^{\circ} \mathrm{C}$ for 48 hours. After incubation, the content of the tubes were decanted and the tubes were washed with Phosphate Buffer Saline (PBS; pH 7) and left to airdry. Subsequently, the tubes were stained with Crystal Violet $(0.1 \% \mathrm{w} / \mathrm{v})$, and the tubes were gently rotated to ensure uniform staining. Afterwards, the stain was removed and tubes were washed with sterile distilled water, and then dried in inverted position. Biofilm formation was considered positive when a visible stained film was observed adhered to the wall and bottom of the tube. Tubes were scored as: 0-absent, 1-weak, 2-moderate or 3 -strong. Experiments were performed in triplicate and repeated three times.

\section{Air liquid interface assay}

The Pseudomonas aeruginosa biofilms were microscopically visualized using Air liquid interface assay [14]. The assay was performed in two different ways. In first experiment, cultures of Pseudomonas aeruginosa were 1:100 diluted in $3 \mathrm{ml}$ Tryptone Soy Broth (TSB; SigmaAldrich). Subsequently, $300 \mu \mathrm{l}$ of each diluted culture was pipetted into each well of a 12-well, flat-bottom plates. The plates were covered with lid and incubate at $37^{\circ} \mathrm{C}$ for 48 hour, in a position that they were making a $45^{\circ}$ angle to the surface of incubator. After incubation, the cultures were aspirated and the wells were gently washed twice by adding $400 \mu \mathrm{l}$ sterile TSB medium. After two washes, $200 \mu \mathrm{l}$ of TSB medium was added to each well. The plate was laid flat on the stage of an inverted microscope (Olympus, Japan) and biofilm formation was visualized. The pictures of the biofilm were taken using a digital camera (Canon-A450, Malaysia).

In the second experiment, cultures of Pseudomonas aeruginosa were 1:100 diluted in $3 \mathrm{ml}$ Tryptone Soy Broth (TSB; Sigma-Aldrich). Subsequently, $300 \mu \mathrm{l}$ of each diluted culture was inoculated in each well of a 12-well plate with cover slips placed at $90^{\circ}$ angle to the well's surface. The 12-well plate was incubated for 48 hours at $37^{\circ} \mathrm{C}$. After incubation, cover slips were taken out, washed with sterile distill water and stained with $0.1 \%$ $\mathrm{CV}$ for 10 minutes. Afterwards, the cover slips were washed and dried. Dry cover slips were visualized under high power microscope (Olympus, Japan) and pictures were taken using a digital camera (Canon-A450, Malaysia).

\section{Microtitre-plate method}

Finally, the Pseudomonas aeruginosa cultures were quantitatively analyzed for their biofilm forming potential using microtitre-plate method [14-16]. Briefly, Pseudomonas aeruginosa cultures were inoculated in $3-5 \mathrm{ml}$ TSB and incubated for 24 hours at $37^{\circ} \mathrm{C}$. After incubation, cultures were 1:100 diluted in the TSB, and $100 \mu \mathrm{l}$ of each diluted culture was pipetted in each well of 96-well flat-bottom 
microtiter plate (non-tissue culture treated, Sigma-Aldrich). Plates was covered and incubated at $37^{\circ} \mathrm{C}$ for 48 hours. After incubation, contents of the well were aspirated out and the wells were washed thoroughly with PBS. Subsequently, the wells were stained for $10 \mathrm{~min}$ by adding $125 \mathrm{ul}$ of $0.1 \%$ Crystal Violet (w/v) solution to each well. Afterwards, the stain was removed and the plate was washed with clean tap water and left to air dry. Subsequently, $200 \mu \mathrm{l}$ of $95 \%$ Ethanol was added to each stained well and plates were incubated for 10 to $15 \mathrm{~min}$ at room temperature. Contents of each well were mixed by pipetting, and then $125 \mu \mathrm{l}$ of the Crystal Violet/Ethanol solution was transferred from each well to a separate well of an optically clear flat-bottom 96-well plate. Optical densities (OD) of each of these 125- $\mu$ l samples were measured at $630 \mathrm{~nm}$ using spectrophotometer (Starfax 2100, Awareness Technology Inc). Experiments were performed in duplicate.

\section{d) Analysis of relationship between biofilm formation and} antibiotic resistance

In order to examine the relationship between forming potential and antibiotic resistance, the antibiotic susceptible and antibiotic resistant isolates were separately grouped and biofilm forming potential of each group was examined. The difference between the groups was analyzed using unpaired two-tailed student's T test using GraphPad software with significance level of $\mathrm{p}<0.05$.

\section{Results}

Isolation of Pseudomonas aeruginosa from contact lenses and contact lens solutions

A total of 22 Pseudomonas aeruginosa isolates were obtained from 36 contact lenses and 14 contact lens solution samples. The cultures were identified on the basis of colony morphology, pigment production and

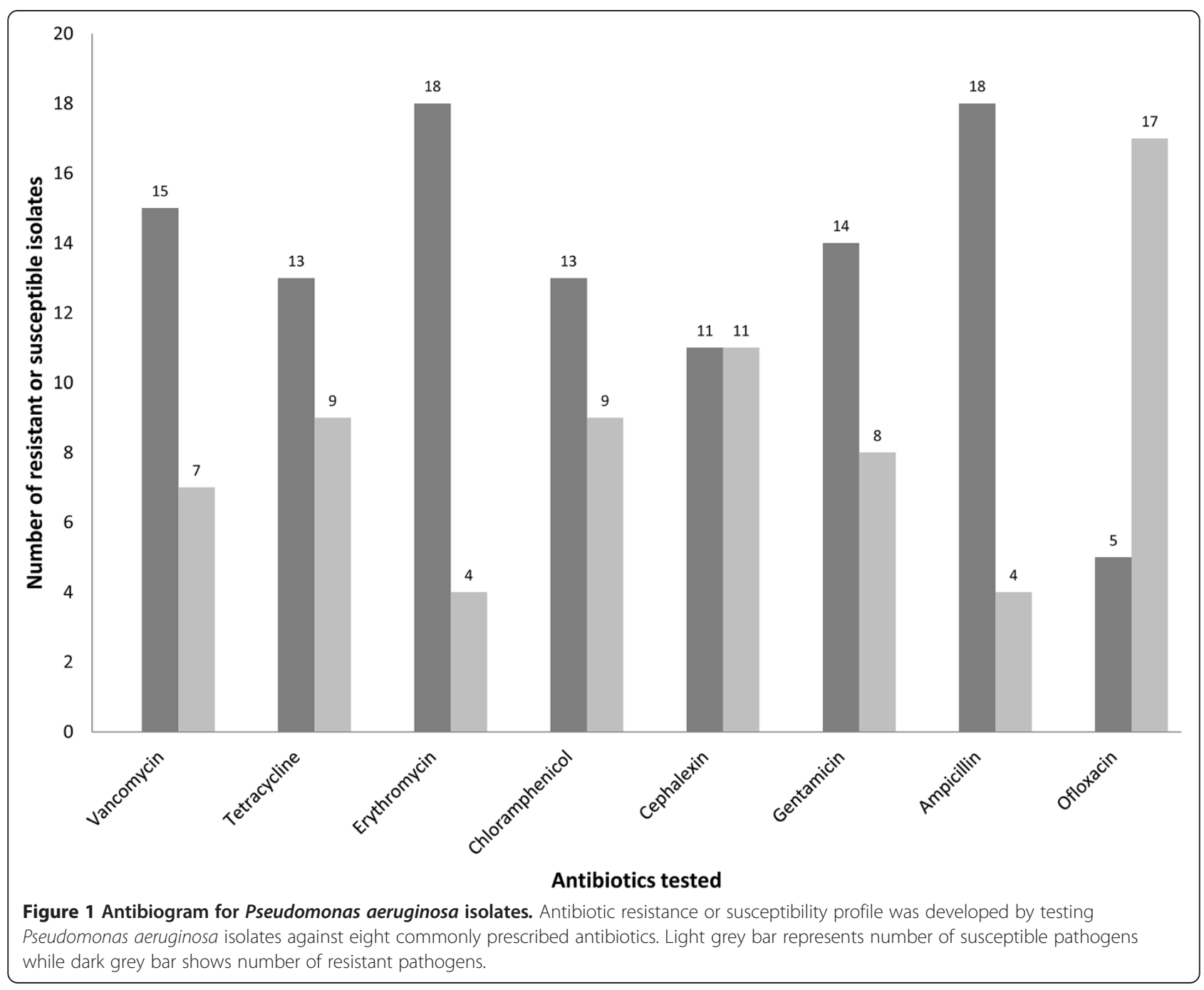


characteristic biochemical reactions using both conventional and rapid identification tests (Quick strip).

\section{Antibiotic resistance/susceptibility profile of Pseudomonas aeruginosa isolates}

The antibiotic resistance/susceptibility profile of Pseudomonas isolates revealed that most of the isolates were resistant to one or more tested antibiotics (Figure 1). Most of the isolates (18/22) were resistant to Erythromycin and Ampicillin (Figure 1). Cephalexin and Ofloxacin were found to be the most effective antibiotics as respectively, $11 / 22$ and $17 / 22$ isolates were susceptible to them (Figure 1).

Biofilm forming potential of Pseudomonas aeruginosa isolates In this study, the biofilm forming potential of Pseudomonas aeruginosa isolates was evaluated using both qualitative (tube method, air liquid interface assay) and quantitative (micro-titre plate assay) methods. In the tube method, Pseudomonas aeruginosa isolates, inoculated in the tubes, formed a dense whitish matt at air-liquid Interface (Figure 2A) and also strongly adhered to the walls of the tubes (Figure 2B). Similarly, the results for the Air liquid assay revealed that the culture of Pseudomonas aeruginosa exhibited dense matt formation (Figure 2C) and strong aggregation (Figure 2D). The quantitative biofilm analysis corroborated the qualitative results, where almost all Pseudomonas aeruginosa isolates were found to be dominant biofilm former with $\mathrm{OD}_{630}>0.5$ (Figure 3).

\section{Relationship between biofilm formation and antibiotic resistance profile}

The statistical analysis to examine the link between antibiotic resistance and biofilm formation showed that the biofilm production in multi-drug resistant isolates was significantly higher that drug susceptible isolates $(\mathrm{P}<0.0001)$.

\section{Discussion}

Pseudomonas aeruginosa has emerged as an important eye pathogen, responsible for serious ophthalmic infections such as keratitis (corneal ulcers) $[17,18]$. Increasing use of contact lens - both for medical or cosmetic use has greatly increased the risk for acquiring Pseudomonal infections [19]. Improper handling and use of contaminated storage solution can pollute the contact lens, which in turn serve as an ideal platform for the bacterial adherence

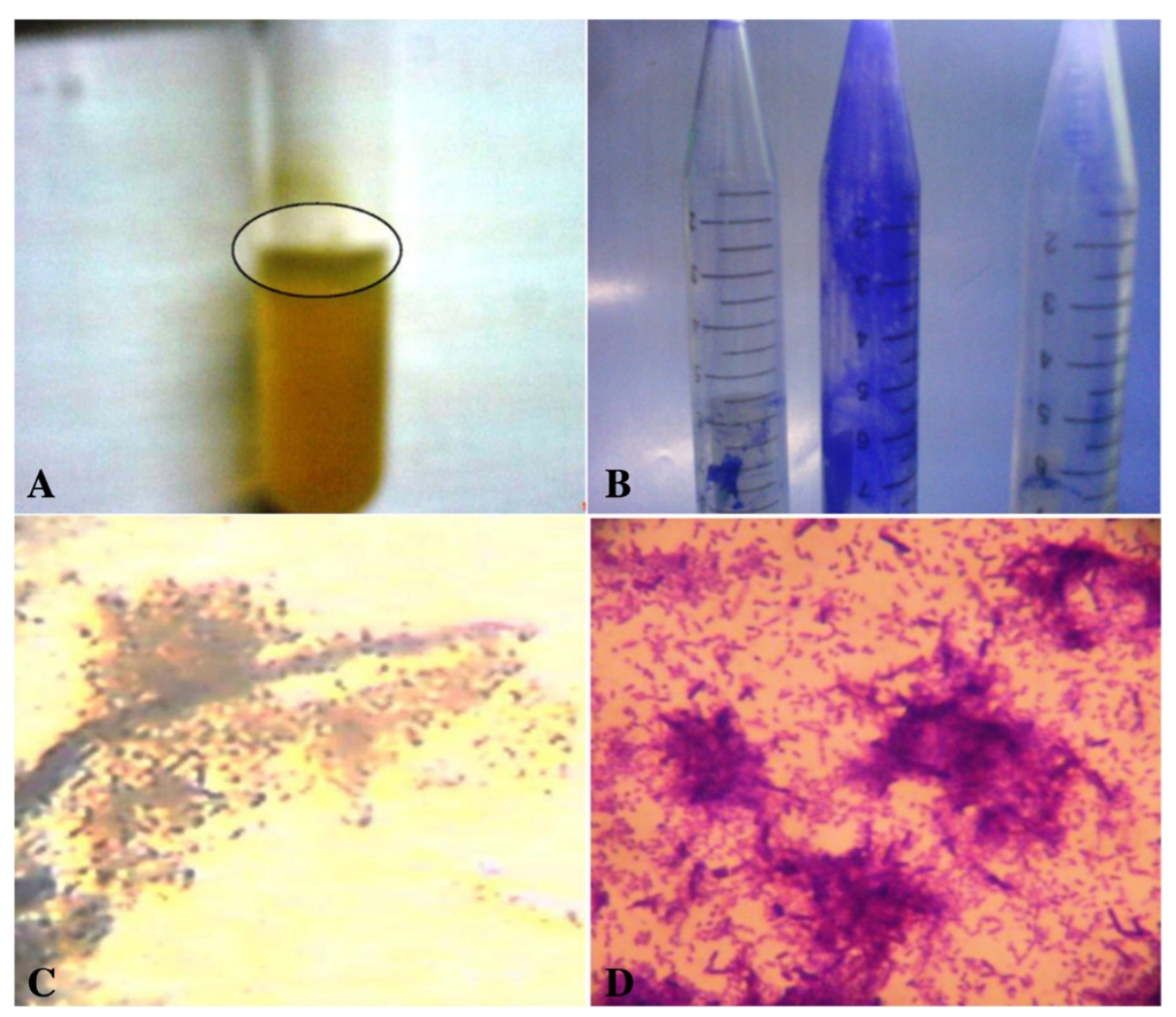

Figure 2 Qualitative analysis of biofilm formation by Pseudomonas aeruginosa. Pictures showing A) dense matt formed at the Air-liquid interface in glass tube (pointed by arrow), B) microorganisms adhered to the surface of polystyrene surface stained with Crystal Violet. A and B) The experiment was performed using tube method. Biofilm formation by Pseudomonas aeruginosa as observed $\mathbf{C}$ ) under inverted microscope using Air-liquid interface assay, D) under compound microscope using Air-Liquid interface cover slip assay. Dense matt formation and microbial aggregation is clearly evident in the $\mathbf{C}$ ) wells and $\mathbf{D}$ ) on the slides. 


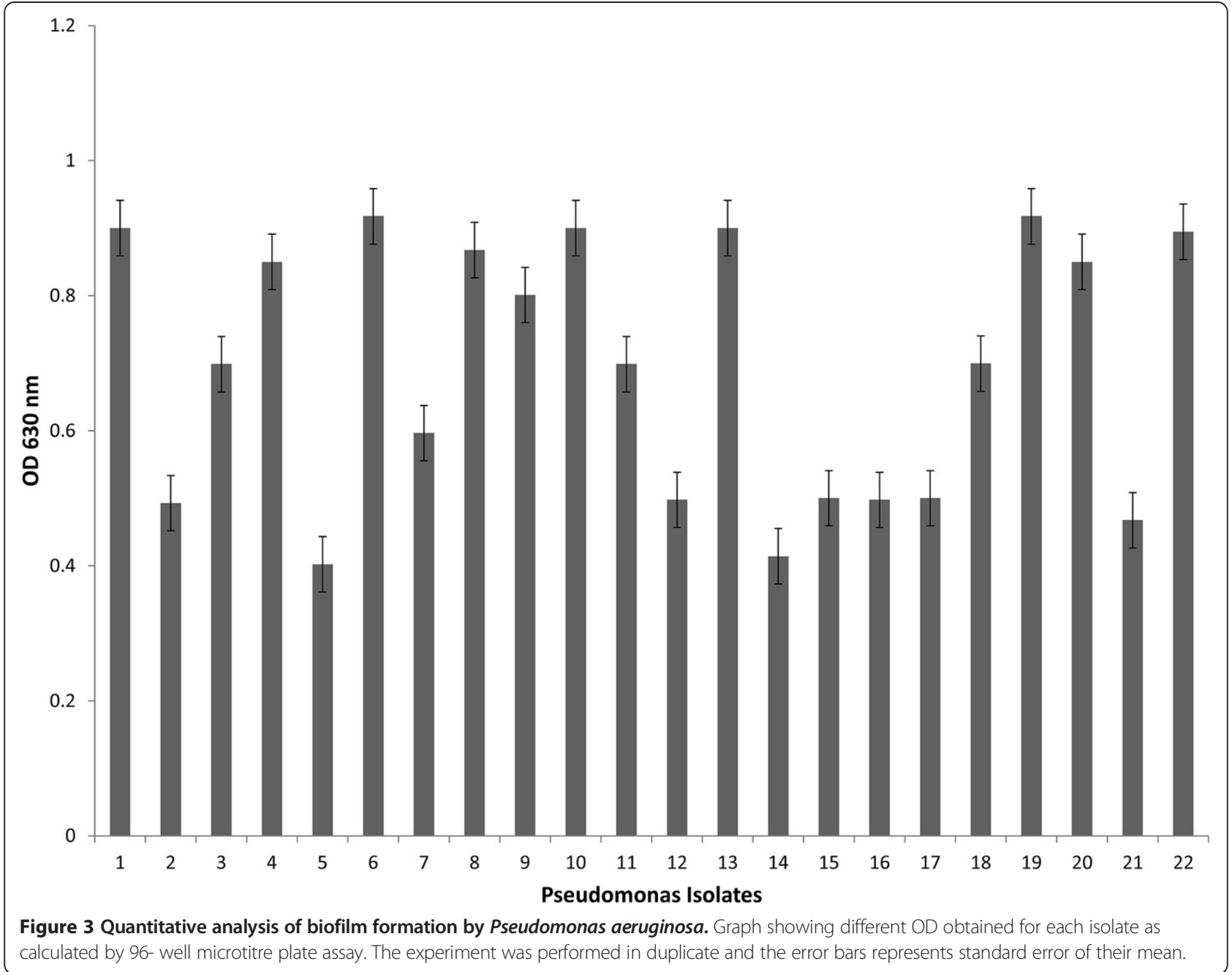

and biofilm production [20,21]. The biofilm forming potential has been associated with increased antibiotic resistance, which ultimately leads to therapeutic failure $[22,23]$. Analysis of the susceptibility/resistance profile our Pseudomonas aeruginosa isolates reveled that majority of them were resistant to one or more tested antibiotics. Pinna et al., and Ly et al., found Aminoglycosides and Fluoroquinolones to be effective in contact lens associated Pseudomonal infections [19,21,24], while Wilcox reported first generation of Cephalosporins with Aminoglycosides as an effective initial treatment in contact lens associated Pseudomonal infections [25]. Our findings were in agreement with these reports, where Pseudomonas isolates exhibited lowest resistance against Ofloxacin (Fluoroquinolone) and Cephalexin (first generation Cephalosporins). However, in contrast to above-mentioned studies, high resistance was observed against Gentamycin (aminoglycoside). In the next step, we analyzed the biofilm forming potential of these isolates, as biofilm plays important role in antimicrobial resistance [26]. Analysis of biofilm formation potential, using both qualitative and quantitative methods, identified almost all Pseudomonas aeruginosa isolates as dominant biofilm formers. Finally, the analysis for relationship between biofilm formation and antibiotic resistance/susceptibility revealed that the multi-drug resistant isolates displayed significant biofilm production as compared to susceptible isolates $(\mathrm{p}<0.0001)$. Our results are consistent with previous reports, where the minimum inhibitory concentration of different antibiotics in different microbial biofilms were found to increase from 10-1000 fold, when compared to non-biofilm forming colonies [27]. This resistance can be explained by the three possible mechanisms: 1) Failure of the antibiotics to penetrate the dense matrix, 2) Sub-optimal concentration of antibiotic, in case the antibiotic penetrates the biofilm, which is below the minimum inhibitory concentration for the microbes inside the biofilm, 3) Inability of the antibiotic to inhibit pathogens, because most the pathogens in deeper layers of biofilm are metabolically inactive, and 4) Expulsion of antibiotics from the biofilm, as a result of cumulative 'efflux action' by the microbial communities [22]. 
We believe our study would serve as a first significant report on Pakistani patients, analyzing the status of antibiotic resistance and biofilm formation in Pseudomonas aeruginosa, isolated from contact lens of patients with different ocular infections. Our results strongly emphasize on the importance of: 1) Proper handling and hygienic storage of contact lens in order to prevent Pseudomonal infections, 2) Selecting correct and effective antimicrobial agents in ocular infections to prevent and control antimicrobial resistance, and 3) Exploring new antimicrobial agents that can target biofilms producing pathogens, especially Pseudomonas aeruginosa, that are involved in contact lens associated infections.

\section{Conclusion}

In conclusion, observing hygienic practices while dealing with contact lenses can save the user from Pseudomonal eye infections. Additionally, careful selection of appropriate antibiotics in contact lens associated infections can not only prevent serious ocular complications, but can also reduce antimicrobial resistance and dissemination of biofilm forming Pseudomonal isolates.

\section{Competing interests}

The authors declared that they have no competing interests.

\section{Authors' contributions}

Study design and analysis: SHA, SKS, SUK. Experiments: SHA, SKS, TRS, AB. Manuscript writing: SHA, SUK. Supervision: SUK. Syed H. Abidi (SHA), Sikandar K. Sherwani (SKS), Tarrunum R. Siddiqui (TRS), Asma Bashir (AB) and Shahana U. Kazmi (SUK). All authors read and approved the final manuscript.

\section{Acknowledgements}

We would like to thank Dr. Mahmooda Kazmi, DESTO-Pakistan, for provision of Quick test strip 24. All consumables were obtained from Immunology and Infectious Diseases Research Laboratory.

\section{Author details}

${ }^{1}$ Immunology and Infectious Diseases Research Lab, Department of Microbiology, University of Karachi, Karachi, Pakistan. ${ }^{2}$ Department of Biological and Biomedical Sciences, Aga Khan University, Stadium Road, Karachi 74800, Pakistan. ${ }^{3}$ Department of Microbiology, Federal Urdu University of Arts, Science and Technology, Karachi, Pakistan. ${ }^{4}$ Akhtar Eye Hospital, and Pakistan Medical Research Council, Karachi, Pakistan.

${ }^{5}$ Department of Biosciences, Shaheed Zulfikar Ali Bhutto Institute of Science and Technology (SZABIST), Karachi, Pakistan.

Received: 11 January 2013 Accepted: 9 October 2013

Published: 17 October 2013

\section{References}

1. Gales AC, Sader HH, Jones RN: Respiratory tract pathogens isolated from patients hospitalized with suspected pneumonia in Latin America: frequency of occurrence and antimicrobial susceptibility profile: results from the SENTRY Antimicrobial Surveillance Program (1997-2000). Diagn Microbiol Infect Dis 2002, 44(3):301-311.

2. Drago L: Bacteria and biofilm in respiratory tract infections. Infez Med 2009, 2(17):3-9.

3. Chi H, Chang KY, Chang HC, Chiu NC, Huang FY: Infections associated with indwelling ventriculostomy catheters in a teaching hospital. Int J Infect Dis 2009, 14(3):e216-219.

4. Branski LK, Al-Mousawi A, Rivero H, Jeschke MG, Sanford AP, Herndon DN: Emerging infections in burns. Surg Infect (Larchmt) 2009, 10(5):389-397.
5. Diec J, Carnt N, Tilia D, Evans V, Rao V, Ozkan J, Holden BA: Prompt diagnosis and treatment of microbial keratitis in a daily wear lens. Optom Vis Sci 2009, 86(7):E904-907.

6. Vijay AK, Sankaridurg P, Zhu H, Willcox MD: Guinea pig models of acute keratitis responses. Cornea 2009, 28(10):1153-1159.

7. Schaefer F, Bruttin O, Zografos L, Guex-Crosier Y: Bacterial keratitis: a prospective clinical and microbiological study. Br J Ophthalmol 2001, 85(7):842-847.

8. Cheng KH, Leung SL, Hoekman HW, Beekhuis WH, Mulder PG, Geerards AJ, Kijlstra A: Incidence of contact-lens-associated microbial keratitis and its related morbidity. Lancet 1999, 354(9174):181-185.

9. DiGaetano M, Stern GA, Zam ZS: The pathogenesis of contact lensassociated Pseudomonas aeruginosa corneal ulceration. // Animal Model Cornea 1986, 5(3):155-158.

10. Lau LI, Wu CC, Lee SM, Hsu WM: Pseudomonas corneal ulcer related to overnight orthokeratology. Cornea 2003, 22(3):262-264.

11. Zegans ME, Becker HI, Budzik J, O'Toole G: The role of bacterial biofilms in ocular infections. DNA Cell Biol 2002, 21(5-6):415-420.

12. Bauer AW, Kirby WM, Sherris JC, Turck M: Antibiotic susceptibility testing by a standardized single disk method. Am J Clin Pathol 1966, 45(4):493-496.

13. Christensen GD, Simpson WA, Bisno AL, Beachey EH: Adherence of slimeproducing strains of Staphylococcus epidermidis to smooth surfaces. Infect Immun 1982, 37(1):318-326.

14. Merritt JH, Kadouri DE, O'Toole GA: Growing and analyzing static biofilms. Curr Protoc Microbiol 2005, 1(1):1.

15. O'Toole G, Kaplan HB, Kolter R: Biofilm formation as microbial development. Annu Rev Microbiol 2000, 54:49-79.

16. O'Toole GA, Pratt LA, Watnick PI, Newman DK, Weaver VB, Kolter R: Genetic approaches to study of biofilms. Methods Enzymol 1999, 310:91-109.

17. Hazlett LD: Corneal response to Pseudomonas aeruginosa infection. Prog Retin Eye Res 2004, 23(1):1-30.

18. Mah-Sadorra JH, Yavuz SG, Najjar DM, Laibson PR, Rapuano CJ, Cohen EJ: Trends in contact lens-related corneal ulcers. Cornea 2005, 24(1):51-58.

19. Mohammadpour M, Mohajernezhadfard Z, Khodabande A, Vahedi P: Antibiotic susceptibility patterns of pseudomonas corneal ulcers in contact lens wearers. Middle East Afr J Ophthalmol 2011, 18(3):228-231.

20. Choo JD, Holden BA, Papas EB, Willcox MD: Adhesion of Pseudomonas aeruginosa to orthokeratology and alignment lenses. Optom Vis Sci 2009, 86(2):93-97.

21. Pinna A, Usai D, Sechi LA, Molicotti P, Zanetti S, Carta A: Detection of virulence factors in Pseudomonas aeruginosa strains isolated from contact lens-associated corneal ulcers. Cornea 2008, 27(3):320-326.

22. Stewart PS, Costerton JW: Antibiotic resistance of bacteria in biofilms. Lancet 2001, 358(9276):135-138.

23. Hoiby N, Bjarnsholt T, Givskov M, Molin S, Ciofu O: Antibiotic resistance of bacterial biofilms. Int J Antimicrob Agents, 35(4):322-332.

24. Ly CN, Pham JN, Badenoch PR, Bell SM, Hawkins G, Rafferty DL, McClellan KA Bacteria commonly isolated from keratitis specimens retain antibiotic susceptibility to fluoroquinolones and gentamicin plus cephalothin. Clin Experiment Ophthalmol 2006, 34(1):44-50.

25. Willcox MD: New strategies to prevent Pseudomonas keratitis. Eye Contact Lens 2007, 33(6 Pt 2):401-403. discussion 410-401.

26. del Pozo JL, Patel R: The challenge of treating biofilm-associated bacterial infections. Clin Pharmacol Ther 2007, 82(2):204-209.

27. Donlan RM, Costerton JW: Biofilms: survival mechanisms of clinically relevant microorganisms. Clin Microbiol Rev 2002, 15(2):167-193.

doi:10.1186/1471-2415-13-57

Cite this article as: Abidi et al:: Drug resistance profile and biofilm forming potential of Pseudomonas aeruginosa isolated from contact lenses in Karachi-Pakistan. BMC Ophthalmology 2013 13:57. 